\title{
A Case of Incus Vibroplasty: Postoperative Changes in Residual Hearing
}

\author{
Kyung Jin Roh, Eun Jung Lee, Byung II Choi, and Eun Jin Son \\ Department of Otorhinolaryngology, Yonsei University College of Medicine, Seoul, Korea
}

$\begin{array}{ll}\text { Received } & \text { June 20, 2014 } \\ \text { Revised } & \text { November 1, 2014 } \\ \text { Accepted } & \text { November 23, } 2014\end{array}$

Address for correspondence Eun Jin Son, MD, PhD

Department of Otorhinolaryngology, Yonsei University College of Medicine,

Gangnam Severance Hospital,

211 Eonju-ro, Gangnam-gu,

Seoul 135-720, Korea

Tel +82-2-2019-3460

Fax +82-2-3463-4750

E-mail ejson@yuhs.ac
In patients with mild to severe hearing loss, conventional hearing aids offer limited benefits and several problems with feedback and cosmesis. Middle ear implants are a feasible option for patients with moderate to severe hearing loss who are unable to achieve adequate benefit from or cannot tolerate hearing aids for various reasons. Here we present a case of middle ear implant surgery using Vibrant Soundbridge with incus vibroplasty technique, and describe the hearing changes during postoperative follow-up. J Audiol Otol 2015;19(1):54-57

KEY WORDS: Middle ear implant · Hearing loss.

\section{Introduction}

Although conventional hearing aids can provide ample benefit for patients with mild to severe hearing loss, patients experience several problems including acoustic feedback, occlusion effect, discomfort, infection, and unsatisfactory cosmesis. Active middle ear implants (AMEIs) may offer adequate amplification without these problems $[1,2]$. The Vibrant Soundbridge (VSB, Med-El, Innsbruck, Austria) is a partially implantable AMEI device comprised of an internal component that utilizes a vibrating ossicular prosthesis (VORP) and an external component with microphone, audio processor, battery, and transmitter coil. Sound information is collected and processed in the audio processor and delivered transcutaneously to the VORP. The transmitted electromagnetic signals are transformed to vibration energy that drives the floating mass transducer (FMT) by an electrical conduit. The VORP can be coupled to the ossicular chain (usually the incus, stapes, or footplate) or to the round window [3-5]. Reports of au-

This is an Open Access article distributed under the terms of the Creative Commons Attribution Non-Commercial License (http://creativecommons. org/licenses/by-nc/3.0/) which permits unrestricted non-commercia use, distribution, and reproduction in any medium, provided the original work is properly cited. diological results and patient satisfaction are generally favorable for AMEI [2,6-9]. Studies have shown that functional gain and speech recognition scores were stable, and that subjective satisfaction with the AMEI was comparable to conventional hearing aids over a long term period of time [10,11]. Despite the expectation that the residual hearing is preserved and better outcomes with AMEI, patients undergoing the AMEI surgery may be distressed during the immediate postoperative period while the implanted ear is temporarily deprived of hearing amplification. In this case report, we describe the changes of the postoperative audiologic outcomes in a case of AMEI using incus vibroplasty over 11 months postoperatively.

\section{Case Report}

A 68-year-old man presented to our department with decreased hearing acuity in both ears. The patient had a longstanding history of chronic otitis media and had undergone tympanoplasty on the right side 8 years prior. He had been using conventional hearing aids in both ears on and off for 8 years. His main issues with the hearing aids were dissatisfaction with the quality of sound, difficulty with speech discrimination, and intermittent skin problems in the external auditory canal. Pure tone audiometry confirmed bilateral sensorineural hear- 
ing loss (SNHL) with increased thresholds at $3 \mathrm{kHz}$ and higher (Fig. 1). Speech discrimination scores for two-syllable words were $72 \%$ in the right ear and $76 \%$ in the left, at presentations level of $70 \mathrm{~dB}$. Temporal bone computed tomography scans showed no active inflammatory changes, and the ossicular chain appeared intact in both ears. During counseling for hearing rehabilitation, the option of using VSB was discussed with the patient. Since he preferred using a hearing aid in the left ear, surgery for VSB was performed in the right ear. Intraoperatively, after mastoidectomy was performed to preserve the posterior external auditory canal wall, a facial recess was opened, and the long process of the incus was identified. Extra care was taken to widen the inferior portion of the facial recess to allow unhindered entrance of the FMT and the specialized forming forceps. Ossicular chain mobility was intact. After the VORP was secured in the bony well, the FMT was introduced into the middle ear cavity. The clip of the FMT was gently placed on the long process of the incus without any excess pressure to the ossicular chain. The axis of the FMT was aligned parallel to the axis of motion of the stapes, and care was taken to ensure that the FMT was close enough to the incudostapedial joint but did not touch the promontory or the pyramidal eminence. Then, the forming forceps were brought through the facial recess with the jaws opened. The forming forceps were centered exactly over the FMT clip, and then the jaws were closed to form the clip over the long process of the incus. The jaws were then carefully opened, and the forceps were retracted without causing trauma to the ossicles (Fig. 2). A sharp pick was used to check the attachment of the clip to the incus, ensuring it was secure enough to hold the FMT in position. Intraoperative Auditory Nerve Response Telemetry (Med-El, Innsbruck, Austria) confirmed

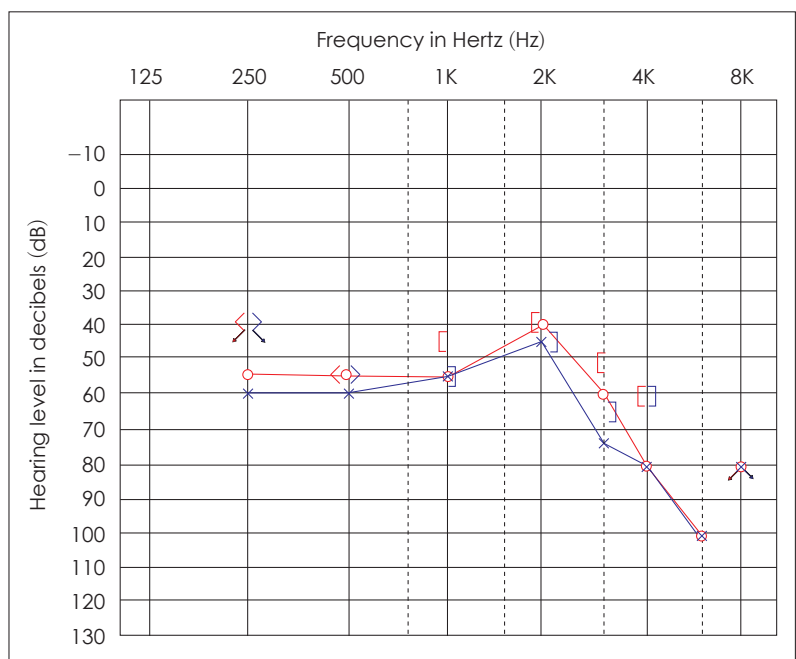

Fig. 1. Preoperative pure tone audiogram shows bilateral sensorineural hearing loss. the positioning of the FMT, and immediate postoperative transocular orbital view confirmed the implanted device (Fig. 3). Audiogram showed increased thresholds at 250 and 500 $\mathrm{Hz}$ at 8 weeks after the surgery, and with the initial mapping, a functional gain of 20-30 dB was achieved (Fig. 4A). During the following 6 months, the air-bone gap noted in the lower frequencies resolved, and the hearing thresholds returned to preoperative levels (Fig. 4B). At 11 months postoperatively, residual hearing was preserved, and the functional gain had increased to $30-40 \mathrm{~dB}$ at 2 and $4 \mathrm{kHz}$ (Fig. 4C). Furthermore, the patient reported better sound quality and more natural voice perception.

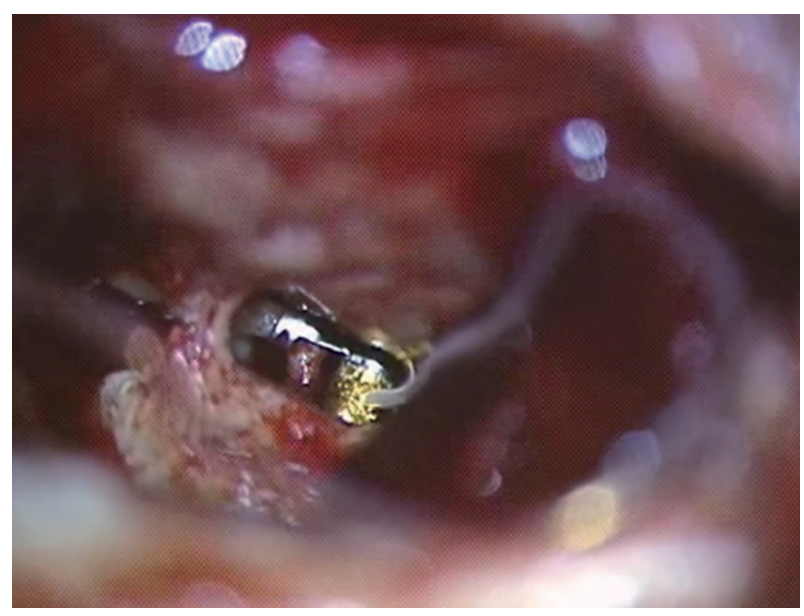

Fig. 2. Intraoperative photo shows the floating mass transducer of the middle ear implant is fixed onto the long process of the incus.

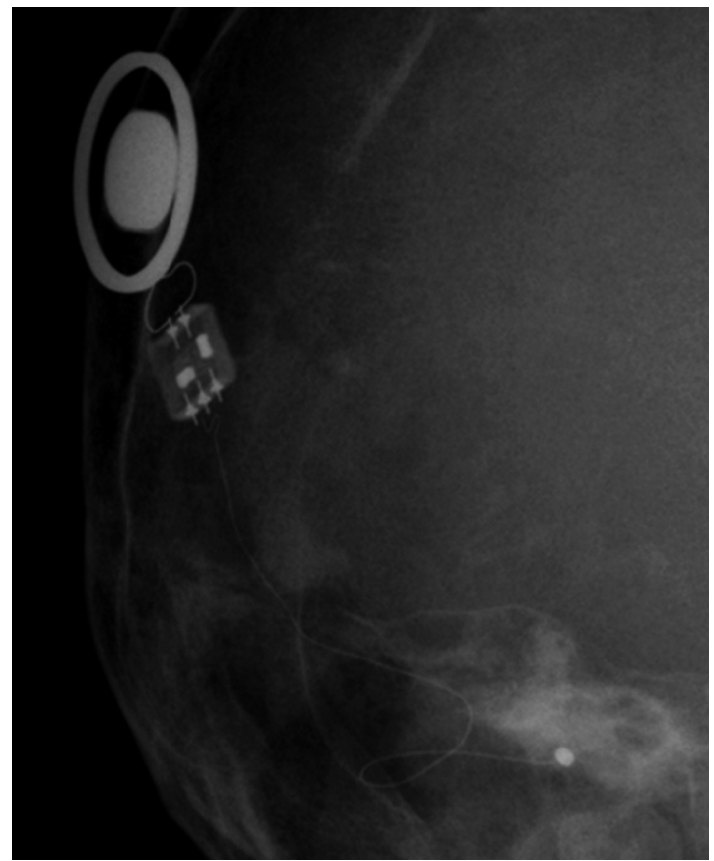

Fig. 3. Immediate postoperative transocular orbital view confirmed the positioning of the implanted device. 

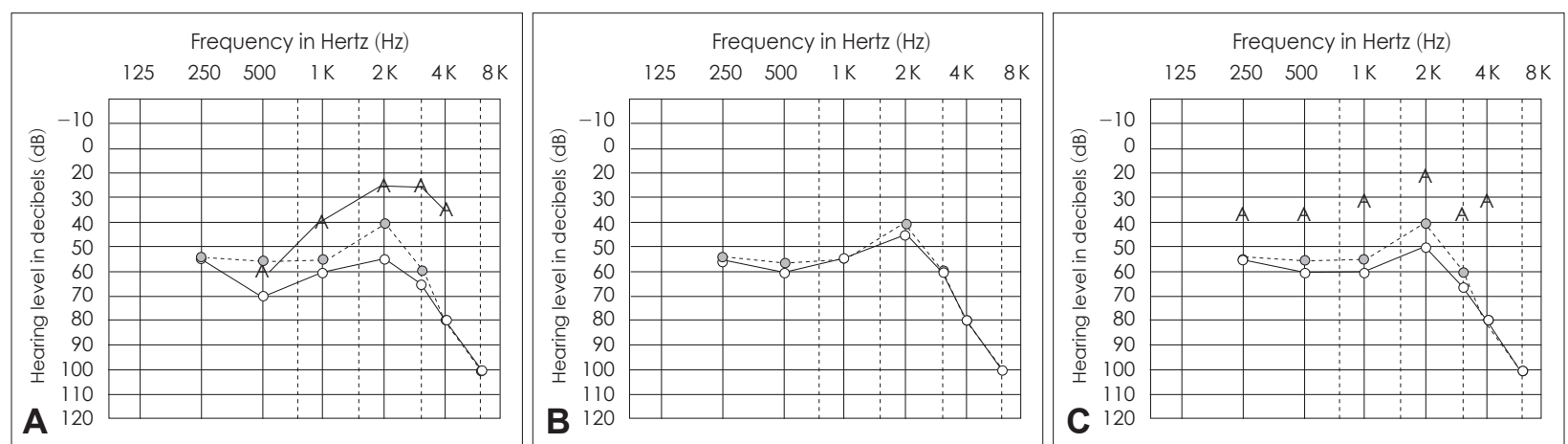

Fig. 4. A: At initial mapping, a functional gain of $20-30 \mathrm{~dB}$ was achieved. B: During the following 6 months, the air-bone gap noted in the lower frequencies resolved, and hearing thresholds returned to preoperative levels. C: At 11 months postoperatively, residual hearing was preserved and functional gain of 30-40 dB was achieved at 2 and $4 \mathrm{kHz}$.

\section{Discussion}

Hearing loss is one of the most prevalent chronic conditions, and prevalence increases with age. However, a significant portion of candidates are not able to tolerate conventional hearing aids. Acoustic feedback, occlusion effect, frequent battery changes, discomfort, infection, and cosmetic problems are among the numerous reasons for low compliance. AMEIs offer several advantages for patients with SNHL who cannot tolerate conventional hearing aids, because the device is semiimplantable and the implanted magnetic actuator directly stimulates the ossicles in the middle ear. One of the concerns patients express when presented with the options for hearing rehabilitation is whether their residual hearing can be preserved after implantation surgery. Audiological outcomes of VSB have been reported to be favorable, and the functional gain of the VSB remained stable postoperatively $[8,10,12,13]$. Residual hearing in the implanted ears was comparable to the contralateral ears, and the functional gain remained stable over $5-8$ years after surgery [11]. Likewise, residual hearing was relatively stable for 8 months in our patient with no significant change.

Incus vibroplasty involves handling of the normally functioning ossicular chain and carries the potential of damage to the ossicles or the cochlear duct. In contrast to conventional cochlear implantees whose residual hearing is minimal, it is important for the candidates of middle ear implants to retain their residual hearing ability without their devices. Thus, utmost care must be taken during the implant surgery. During mastoidectomy, damage to the ossicles, usually the incus, should be avoided. In order to obtain adequate working space for the forming forceps, the facial recess needs to be widened. When using small burrs to widen the superior margin of the facial recess, direct trauma to the incus or the incudostapedial joint should be avoided. Before the FMT is introduced into the middle ear cavity, it is advisable to use both the FMT siz- er and the forming forceps to ensure that working space within the facial recess is adequate for the following procedures. The most important step of incus vibroplasty is securing the attachment clip on the long process of the incus, which may cause significant trauma. The surgeon's hand should be kept steady and excess pressure must be avoided when closing the crimp on the incus. Also, repeated manipulation of the FMT should be avoided. The FMT must be positioned parallel to the stapes but not contacting the promontory. The role of perioperative glucocorticoids has been described for cochlear implant operations, and they may be helpful for middle ear implant operations as well. During the postoperative period, the hearing thresholds were elevated but returned to initial levels at switch-on about 6 months postoperatively. This transient air-bone gap could partially be attributed to blood collection or the use of gelfoam and fibrin glue in the middle ear cavity. Delay in formation of fibrotic adhesion between the FMT and the incus long process may be another factor. Previous studies have ascribed postoperative hearing deterioration to middle ear problems or injury to the ossicles $[1,13]$. Initial mapping is usually recommended about 8 weeks after the surgery to allow a secure connection between the FMT and the ossicular chain. In our patient, the air-bone gap persisted up to 6 months although adequate gain could be achieved through the device. A possible explanation may be that surgical trauma resulted in temporary hypermobility of the ossicular chain causing the air-bone gap, but the direct drive of FMT was sufficient to overcome the burden. In summary, although an increased air-bone gap in residual hearing persisted for up to 6 months, residual hearing could be preserved long-term after the incus vibroplasty technique.

\section{Acknowledgments}

This work is supported by a faculty research grant of Yonsei University College of Medicine (6-2012-0023) to E.J.S. 


\section{REFERENCES}

1) Tysome JR, Moorthy R, Lee A, Jiang D, O'Connor AF. Systematic review of middle ear implants: do they improve hearing as much as conventional hearing AIDS? Otol Neurotol 2010;31:1369-75.

2) Fisch U, Cremers CW, Lenarz T, Weber B, Babighian G, Uziel AS, et al. Clinical experience with the Vibrant Soundbridge implant device. Otol Neurotol 2001;22:962-72.

3) Tringali S, Koka K, Deveze A, Holland NJ, Jenkins HA, Tollin DJ. Round window membrane implantation with an active middle ear implant: a study of the effects on the performance of round window exposure and transducer tip diameter in human cadaveric temporal bones. Audiol Neurootol 2010;15:291-302.

4) Lupo JE, Koka K, Hyde BJ, Jenkins HA, Tollin DJ. Physiological assessment of active middle ear implant coupling to the round window in Chinchilla lanigera. Otolaryngol Head Neck Surg 2011;145:641-7.

5) Monini S, Filippi C, Atturo F, Barbara M. Is the Bone-Conduction HeadBand test useful for predicting the functional outcome of a round window active middle ear implant? Otol Neurotol 2013;34:1329-35.

6) Truy E, Philibert B, Vesson JF, Labassi S, Collet L. Vibrant soundbridge versus conventional hearing aid in sensorineural high-frequency hearing loss: a prospective study. Otol Neurotol 2008;29:684-7.

7) Zwartenkot JW, Hashemi J, Cremers CW, Mulder JJ, Snik AF. Active middle ear implantation for patients with sensorineural hearing loss and external otitis: long-term outcome in patient satisfaction. Otol Neurotol 2013;34:855-61.

8) Verhaegen VJ, Mulder JJ, Cremers CW, Snik AF. Application of active middle ear implants in patients with severe mixed hearing loss. Otol Neurotol 2012;33:297-301.

9) Bernardeschi D, Hoffman C, Benchaa T, Labassi S, Beliaeff M, Sterkers O, et al. Functional results of Vibrant Soundbridge middle ear implants in conductive and mixed hearing losses. Audiol Neurootol 2011;16:381-7.

10) Sterkers O, Boucarra D, Labassi S, Bebear JP, Dubreuil C, Frachet B, et al. A middle ear implant, the Symphonix Vibrant Soundbridge: retrospective study of the first 125 patients implanted in France. Otol Neurotol 2003;24:427-36.

11) Schmuziger N, Schimmann F, àWengen D, Patscheke J, Probst R. Long-term assessment after implantation of the Vibrant Soundbridge device. Otol Neurotol 2006;27:183-8.

12) Vincent C, Fraysse B, Lavieille JP, Truy E, Sterkers O, Vaneecloo FM. A longitudinal study on postoperative hearing thresholds with the Vibrant Soundbridge device. Eur Arch Otorhinolaryngol 2004;261: 493-6.

13) Mosnier I, Sterkers O, Bouccara D, Labassi S, Bebear JP, Bordure P, et al. Benefit of the Vibrant Soundbridge device in patients implanted for 5 to 8 years. Ear Hear 2008;29:281-4. 